\title{
A multidisciplinary team case management approach reduces the burden of frequent asthma admissions
}

\author{
Hannah Burke, Jenny Davis, Sian Evans, Laura Flower, Andrew Tan and \\ Ramesh J. Kurukulaaratchy
}

Affiliation: Dept of Respiratory Medicine, University Hospital Southampton NHS Foundation Trust, Southampton, UK.

Correspondence: Ramesh J. Kurukulaaratchy, Dept of Respiratory Medicine, Mailpoint 52 G-level West Wing, University Hospital Southampton NHS Foundation Trust, Tremona Road, Southampton, S016 6YD, UK.

E-mail: R.J.Kurukulaaratchydsoton.ac.uk

ABSTRACT Up to $10 \%$ of asthmatics have "difficult asthma"; however, they account for $80 \%$ of asthmarelated expenditure and run the highest risk of acute severe exacerbations. An estimated $75 \%$ of admissions for asthma are avoidable. Guidelines advise that these patients be managed by an experienced specialist multidisciplinary team (MDT). We aimed to assess the impact of a case management strategy delivered via specialist MDTs on acute healthcare utilisation of patients with frequent asthma admissions.

An MDT (consultant, specialist nurse, physiotherapist and psychologist) case management strategy was introduced in 2010 at University Hospital Southampton Foundation Trust (Southampton, UK) to support patients with frequent asthma admissions during admission and then in clinic. To assess efficacy, we systematically searched the hospital database for patients acutely admitted for asthma on two or more occasions in 2010, 2011 and 2012. Data were collected retrospectively covering patient demographics, admission details, asthma severity and comorbidity.

From 2010 to 2012, 84 patients were admitted on two or more occasions per year ( $80 \%$ female, mean body mass index $31 \mathrm{~kg} \cdot \mathrm{m}^{-2}$ and $55 \%$ psychological comorbidity). After introducing an MDT approach repeat asthma admissions fell by 33\% from 127 in 2010 to 84 in 2012 ( $p=0.0004$ ). In addition, bed days fell by $52 \%$ from 895 in 2010 to 430 in 2010 ( $p=0.015$ ).

An MDT case management approach significantly reduces hospitalisation in difficult asthma patients with prior frequent admission.

@ERSpublications

MDT case management approach significantly reduces hospitalisation in asthma patients with prior frequent admission http://ow.ly/7iOI3016277

Received: March 212016 | Accepted after revision: June 012016

Conflict of interest: None declared.

The content of this work is @ the authors or their employers. Design and branding are @ERS 2016. This article is open access and distributed under the terms of the Creative Commons Attribution Non-Commercial Licence 4.0. 


\section{Introduction}

The prevalence of asthma in the United Kingdom (UK) ranks among the highest globally, and consequently the health economic burden of asthma in the UK is considerable [1]. Thus, in the UK, asthma accounts for $>65000$ hospital admissions per year with an annual spend of $£ 800$ million on pharmaceutical costs alone $[1,2]$. Up to $5-10 \%$ of asthmatics have "difficult asthma", defined by the British Thoracic Society guidelines [3] as persistent symptoms and/or frequent exacerbations despite step 4 or 5 treatment. Such patients are 20 times more likely to be admitted to hospital [4], account for $50 \%$ of asthma-related expenditure and run the highest risk of acute severe exacerbations [5]. The recent National Review of Asthma Deaths report [2] highlighted the importance of recognising hospitalisation as a predictor of mortality, with $47 \%$ of patients who died of asthma having experienced a prior hospital admission at some point.

Asthma is a heterogeneous disease composed of numerous phenotypes, and phenotype-specific therapies promise enhanced treatment success. Several approaches have been used to characterise asthma subgroups including the Severe Asthma Research Program (SARP) study [6], which identified five asthma subphenotypes using unbiased cluster analysis, including a later onset, non-Th2 obesity related phenotype seen in females with a high symptom burden (cluster 3). Asthma is frequently associated with aggravating comorbidities, including allergic rhinosinusitis, gastro-oesophageal reflux disease (GORD) and obesity. These comorbidities may further influence the asthma phenotype, affecting underlying pathophysiological processes, and/or acting as confounding factors in the diagnosis or assessment of control of asthma. The prevalence of comorbidities seems to be higher in patients with difficult asthma, and may be particularly detrimental to asthma control in these individuals [7, 8]. For example, TEN BRINKE et al. [8] reported that severe nasal sinus disease, GORD, recurrent respiratory infections, psychological dysfunction and obstructive sleep apnoea were all independently associated with frequent exacerbations in patients with difficult asthma.

Optimal management of patients with difficult asthma is reliant upon rigorous systematic multidisciplinary assessment. This approach facilitates confirmation of an asthma diagnosis as well as an opportunity to identify alternative or concurrent diagnoses that may be contributing to persisting symptoms [4]. To achieve this, patients with difficult asthma often require support from tertiary-level specialist services which provide care from a multidisciplinary team (MDT), comprising specialist medical input, psychological support, physiotherapy input and social care with ready access to allied expertise such as allergy services [3]. Case management is a strategy being developed across the UK health service as a tool for improving care for patients with long-term conditions. It is defined as the process of planning, coordinating, managing and reviewing the care of an individual [9]. One of the priorities of this approach is to provide intensive, personally tailored care to the 3-5\% of people at greatest risk of hospital admission. The evidence for case management is mixed; however, when implemented effectively it has improved the experiences of patients and healthcare providers [10], supported better healthcare outcomes [11] and reduced the utilisation of hospital-based services [12]. Factors important in achieving successful outcomes in case management include assigned accountability of a team to the patients being case managed, accurate case finding to ensure interventions target patients with defined case needs, empowering patients to manage their own condition and continuity of care to reduce the risk of unplanned admission to hospital [13]. Limited research exists into using this strategy in delivering asthma care; however, the King's Fund report [14] recommends that commissioning bodies should clarify the needs of their at-risk population and then consider how to organise services to address these needs using a case management approach.

A subgroup of patients with difficult asthma suffer frequent asthma exacerbations and experience repeated hospital admissions. They pose a particular challenge that will be familiar to healthcare providers regardless of geographical location, which we sought to understand better in our own institution in 2010 [15]. Having identified the nature of the problem we subsequently implemented a case management driven MDT approach to manage this group of repeatedly admitted patients with asthma. In this article we report the outcomes of this novel management pathway over a 3-year monitoring period.

\section{Methods}

In 2010, we identified a cohort of adult patients with known asthma with high healthcare usage at University Hospital Southampton Foundation Trust (UHSFT) (Southampton, UK). A systematic retrospective search of the hospital database identified adult patients with known asthma (aged $\geqslant 18$ years) who had been acutely admitted with asthma on two or more occasions. Four authors (HB, JD, SE and AT) independently reviewed patient electronic and paper records, collecting data on patient demographics, admission details, asthma severity and comorbidity. Any discrepancies were resolved by rechecking the primary source documentation. These patients all had a known prior diagnosis of asthma. That diagnosis of asthma was confirmed during the case management process by reviewing the clinical history along with the patient's lung function results, including bronchodilator reversibility, peak flow diurnal variability or 
histamine bronchial challenge, where appropriate. Psychological comorbidity was recorded if the patient had a diagnosis of anxiety, depression or psychotic disorder in their general practitioner records or if they were taking anxiolytic, antidepressant or antipsychotic medication. Vocal cord dysfunction was diagnosed by suggestive clinical history and examination (auscultation of glottic wheeze) findings. A clinical diagnosis of dysfunctional breathing was based on a combination of clinical history, examination and Nijmegen score. Diagnoses of salicylate sensitivity, GORD and allergic rhinitis were clinically based using standard defining criteria. High-resolution computed tomography scans were reviewed (if available) for concurrent diagnoses of bronchiectasis or emphysema. Coding data were analysed to determine admission costs. Data collection was performed as part of a clinical service review of the difficult asthma service at UHSFT, and therefore formal ethical approval was not sought.

Results of this initial assessment in 2010 have been reported previously [15]. In summary, patients admitted on two or more occasions accounted for a third of all asthma admissions in 2010. They were predominantly female and commonly had psychological comorbidity (69.4\%). Patients with psychological comorbidity showed significantly higher body mass index (BMI) ( $\mathrm{p}=0.012)$, prevalence of dysfunctional breathing $(\mathrm{p}=0.012)$ and trends for greater length of stay. The annual cost of repeated asthma admission was $£ 226536$, of which $72.7 \%$ was accounted for by patients with psychological comorbidity.

In response to these findings we sought to reduce frequent asthma admissions by introducing a specialist nurse coordinated asthma MDT pathway to case manage such patients both during hospitalisation and subsequent clinic follow-up (summarised in fig. 1). If the patient had been admitted with acute asthma in the previous 12 months, was known to the difficult asthma clinic or admitted to a high dependency area, they were triaged to an inpatient team led by a difficult asthma specialist consultant (RJK) with allergy expertise. Otherwise they were managed under a general respiratory team with specialist asthma nurse support. In addition to consultants and nurses, MDT support included a dedicated physiotherapist and a newly appointed clinical psychologist to provide psychological input to address the high prevalence of psychological morbidity among the frequently admitted asthma population at UHSFT. Upon discharge, patients were followed-up in either the asthma nurse led-clinic or difficult asthma clinic, depending on clinical need.
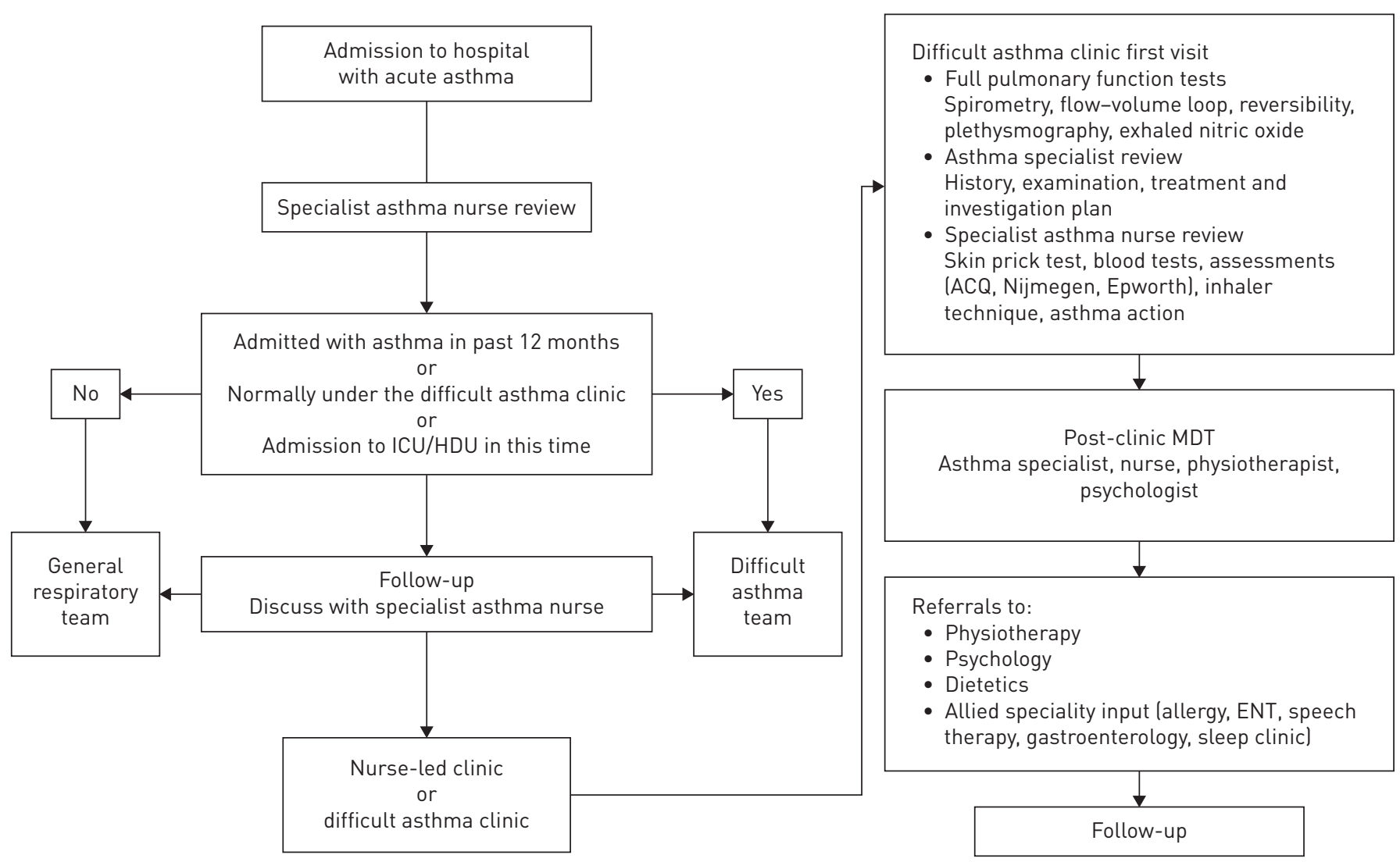

FIGURE 1 Summary of the specialist nurse coordinated multidisciplinary team (MDT) pathway at University Hospital Southampton Foundation Trust, Southampton, UK. ICU: intensive care unit; HDU: high-dependency unit; ACQ: asthma control questionnaire; ENT: ear, nose and throat. 
To assess the efficacy of this intervention within a service evaluation framework, we repeated the systematic search of the hospital database for patients who had been admitted on two or more occasions in 2011 and 2012 and compared the results to 2010. Our main outcome measures assessed the burden of frequently admitted patients with asthma on hospital admissions and included length of stay (total number of bed days per year) as well as admission costs. Results were analysed using standard nonparametric statistical methods to compare the outcomes over a 3-year period.

\section{Results}

Between 2010 and 2012, 103 patients were admitted with acute asthma to UHSFT on two or more occasions per year, comprising 36 patients in 2010, 36 in 2011 and 31 patients in 2012 (fig. 2). These patients were not admitted to any other hospitals with acute asthma during the study period. Only two patients were consecutively admitted on two or more occasions in 2010, 2011 and 2012, and each year the service identified $\geqslant 26$ new frequently admitted patients with asthma who benefited from intensive specialist asthma input. Nevertheless, each year this group showed consistent features: patients were predominantly female, obese and had numerous comorbidities (table 1).

After introducing an MDT approach in 2011, the total number of repeat asthma admissions fell by 33\% from 127 per year in 2010 to 84 in 2012 ( $p=0.0004$ ). In addition, bed days fell by 52\% from 895 per year in 2010 to 430 in 2012 ( $\mathrm{p}=0.015$ ). Number of admissions per frequently admitted patient fell significantly from a mean of 3.5 admissions per patient in 2010 to 2.6 admissions per patient in 2012 ( $\mathrm{p}=0.0015)$. In addition, individual bed days were cut by 50\% from 2010 (mean of 24.9 per person) to 2012 (mean of 11.9 per person) ( $\mathrm{p}=0.019$ ) (fig. 3). Based on analysis of coding data for each admission, UHSFT repeated asthma admission costs fell from $£ 226536$ in 2010 to $£ 147781$ in 2012.

\section{Discussion}

In 2010 we identified a subgroup of patients with asthma who were admitted frequently to UHSFT, resulting in a significant burden on hospital resources. Many had features of difficult asthma including a number of aggravating comorbidities including GORD, dysfunctional breathing and psychological morbidity. By identifying the needs of our local population we designed and implemented a specialist asthma MDT care pathway to case manage these patients. This included targeting specific comorbidities identified in our patients, for example with the introduction of a clinical psychologist to manage the high prevalence of psychological morbidity in our patients. We found significant reductions in hospital admissions and bed days for frequently admitted patients with asthma after implementing this case management strategy. Although we recognise that our study is part of a retrospective service evaluation, our data represents a "real-life", well-characterised asthma cohort followed over a 3-year period. The benefits of that approach were seen to be sustained and incremental. Therefore, we feel that this approach could have wider implications for management of patients with difficult asthma and would encourage that the lessons learned from this service review can be implemented elsewhere to improve patient outcomes.

FIGURE 2 Number of individual patients admitted with asthma on two or more occasions from 2010 to 2012 .

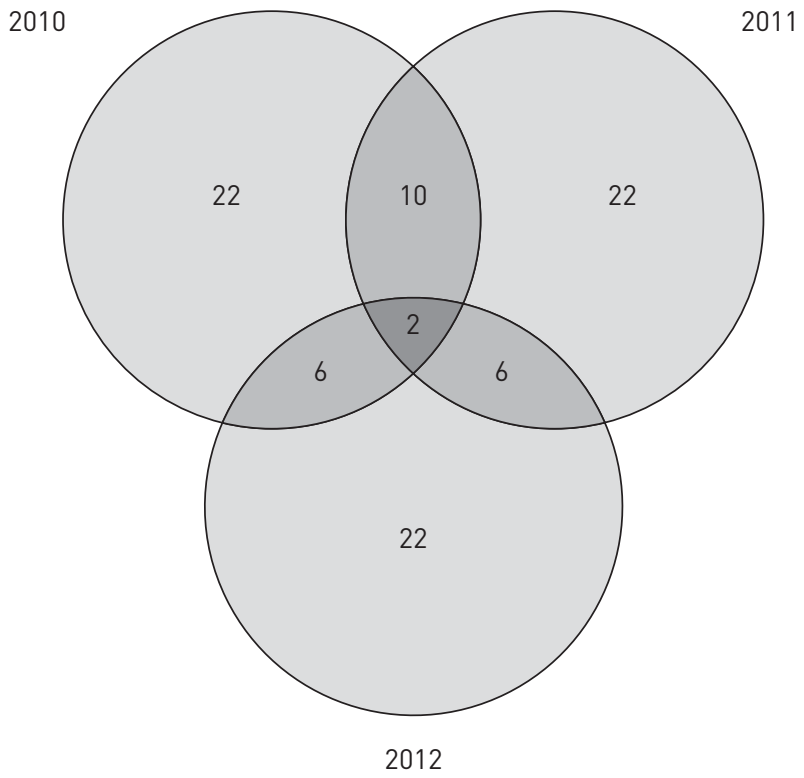




\section{TABLE 1 Characteristics of patients frequently admitted with asthma to University Hospital Southampton Foundation Trust} from 2010 to 2012

\begin{tabular}{|c|c|c|c|c|}
\hline & 2010 & 2011 & 2012 & Total cohort ${ }^{\#}$ \\
\hline Female & 72 & 83 & 81 & 80 \\
\hline FEV 1 L & $2.06(0.64-4.4)$ & $1.8(0.87-3.53)$ & $2.21(0.99-3.46)$ & $2.04(0.64-4.4)$ \\
\hline FEV $1 \%$ & $68.74(26-111)$ & $62.13(47-111)$ & $83.65(46-109)$ & $68.16(26-111)$ \\
\hline FEV $1 /$ FVC & $71.25(34-96)$ & $69.21(32-96)$ & $72.15(27-91)$ & $70.9(27-96)$ \\
\hline GORD & 50 & 44.4 & 54.8 & 46.4 \\
\hline Dysfunctional breathing & 33.3 & 38.9 & 54.8 & 41.7 \\
\hline Allergic rhinitis & 44.4 & 50 & 41.9 & 40 \\
\hline Salicylate sensitivity & 30.6 & 33.3 & 25.8 & 25 \\
\hline Vocal cord dysfunction & 13.9 & 27.8 & 29 & 17.9 \\
\hline Asthma-COPD overlap & 13.9 & 19.4 & $9.7 \%$ & 14.3 \\
\hline
\end{tabular}

Data are presented as \%, mean \pm SD or mean (range), unless otherwise stated. FEV1: forced expiratory volume in 1 s; FVC: forced vital capacity; GORD: gastro-oesophageal reflux disease; COPD: chronic obstructive pulmonary disease; ABPA: allergic bronchopulmonary aspergillosis; BMI: body mass index. ${ }^{\#}$ : excludes patients who were admitted in multiple years; ${ }^{\text {I: }}$ data not available for some patients.

An important part of our intervention was the use of a case management approach. This is an established healthcare tool designed to integrate services around the needs of individuals with long-term conditions [14]. Currently there are only a limited number of studies, mainly based in community practice [16] or paediatric care [17], evaluating the effectiveness of this approach in asthma care. Although it is normally a community-based tool, we have shown effective implementation in secondary care for the management of frequently admitted patients with asthma.

Employing this case management strategy provided a continuity of care that transcended inpatient and outpatient care and facilitated effective carer-patient partnerships. This approach highlights the likely value of continuity of care, which is often emphasised as an important dimension of quality care in primary care, but is not given the same recognition in secondary or tertiary care. Given the complexity of these patients' cases it is certainly worth considering that such continuity of care was a key component in the successful delivery of our care pathway.

We identified $\geqslant 26$ new frequently admitted patients with asthma each year, previously unknown to secondary care. This focuses attention on the potential benefit of even earlier identification in primary care aimed at identifying risk factors for admission that might prevent future admissions. In that vein, RYAN et al. [18] proposed SIMPLES as a structured primary care approach to review uncontrolled asthmatics. Such approaches require close cooperation between primary and specialist care, but could further significantly improve asthma outcomes.
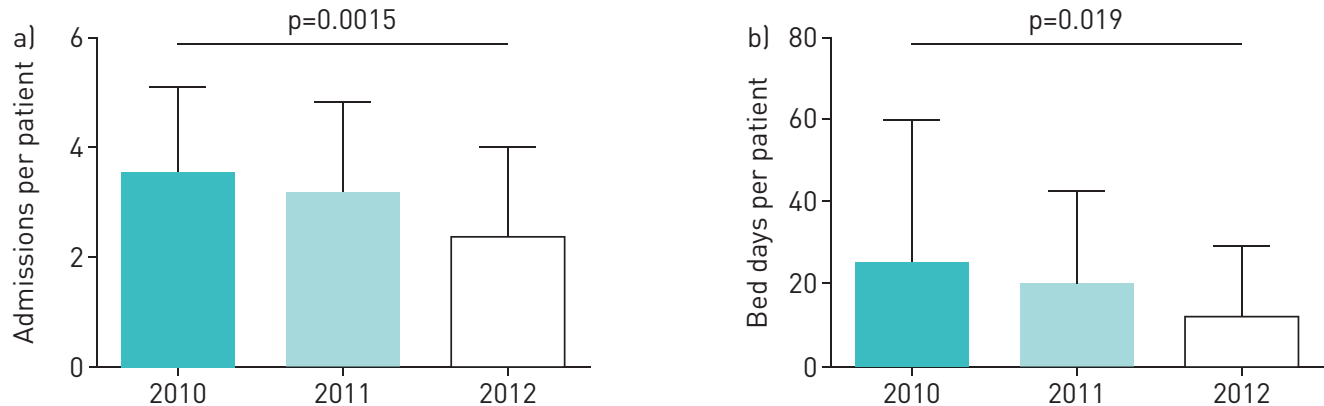

FIGURE 3 Reduction in a) admissions and b) bed days after implementation of a multidisciplinary team case management strategy. Data are presented as mean \pm SD. 
An MDT strategy is key in managing these patients due to the complex multicomponent nature of their health problems. AT UHSFT we manage patients with difficult asthma as part of a "difficult breathing syndrome", defined as a combination of diverse potential asthma phenotypes plus aggravating comorbidities (such as rhinitis, GORD and obesity) and psychosocial factors. By using a systematic approach in addition to phenotype-orientated treatment of airways disease, possible triggers for worsening symptoms are identified and a personalised therapeutic strategy pursued, addressing the whole patient rather than just their airways disease. Allied health professionals play a central role in supporting this model of care. For example, the multidisciplinary input of a specialist asthma nurse has been shown to improve standards of discharge management for acute patients with asthma [19]. Furthermore, dedicated physiotherapy input in the management of dysfunctional breathing in patients with difficult asthma has shown a clinically relevant improvement in quality of life in conjunction with an improvement in Nijmegen scores [20]. However, while assessment for coexistent psychological comorbidity is recommended, there remains limited evidence supporting targeted psychological intervention in patients with difficult asthma, especially when limited to psychological education, rather than a formal psychotherapeutic intervention [21, 22]. Despite this, we have found the introduction of a clinical psychologist into our MDT highly beneficial in supporting better asthma outcomes. Specifically, our clinical psychologist delivers targeted psychotherapeutic interventions using techniques from cognitive behavioural therapy and acceptance and commitment therapy. In addition, they provide education to the wider MDT ensuring all team members engage in psychological support using a consistent and shared approach. Furthermore, although in this study we have used general practitioner records to identify the presence of underlying psychological comorbidity, in clinical practice this is likely to underestimate prevalence. Therefore, our service now adopts a range of screening tools, as well as proactive questioning during consultations to unmask any underlying psychological issues.

The high prevalence of psychological comorbidity in our cohort (55\%) is not unusual in patients with difficult asthma [23]. A recent case series demonstrated similar patient benefits of psychological support strategies for patients with difficult asthma in both our centre and another in the UK [24]. Collectively, these findings highlight the need for larger, well-conducted randomised trials in order to determine the effects of psychological interventions in the management of patients with difficult asthma.

Our results identified our frequently admitted patients as predominantly female (80\%) and obese with dysfunctional breathing and psychological morbidity. This may resemble the older female patients (cluster 3 ) with raised BMI, disproportionately high symptom expression and high healthcare utilisation described in the SARP [6]. HaLdar et al. [25] described a similar phenotype (cluster 2) that was predominantly female, obese and nonatopic. Our data suggest that this relatively small asthma phenotype is responsible for a high number of asthma admissions, and given the resemblance to the phenotypes identified in other studies, may be relevant to other asthma cohorts.

While successful in our hospital, we acknowledge that this case management strategy for frequent asthma admissions may not be readily adoptable in other hospitals. However, we have recently shown that a multidisciplinary approach to difficult asthma care can be successfully delivered away from a specialist centre [26]. If nothing else, this process of developing a local solution to a local problem is to be encouraged and can be used to build required resources. For example, at UHSFT, identifying a high prevalence of psychological morbidity in our frequently admitted patients with asthma in 2010 provided evidence to support successful investment for a clinical psychologist who was key in delivering our MDT asthma care pathway.

We propose that further research should focus on prospective controlled trials to validate this approach in other populations and identify key aspects of the MDT programme that may be universally applied and those that may be population specific.

\section{References}

1 Asthma UK. Asthma Facts and Statistics. www.asthma.org.uk/about/media/facts-and-statistics/ Date last accessed: March 18, 2016. Date last updated: June 1, 2013.

2 Royal College of Physicians. Why Asthma Still Kills: the National Review of Asthma Deaths (NRAD) Confidential Enquiry Report. London, Royal College of Physicians, 2014.

3 British Thoracic Society, Scottish Intercollegiate Guidelines Network. British guideline on the management of asthma. Thorax 2014; 69: Suppl. 1, 1-192.

4 Robinson DS, Campbell DA, Durham SR, et al. Systematic assessment of difficult-to-treat asthma. Eur Respir J 2003; 22: 478-483.

5 National Asthma Education and Prevention Program. Expert Panel Report: Guidelines for the Diagnosis and Management of Asthma Update on Selected Topics. J Allergy Clin Immunol 2002; 110: Suppl. 5, S141-S219.

6 Moore WC, Meyers DA, Wenzel SE, et al. Identification of asthma phenotypes using cluster analysis in the Severe Asthma Research Program. Am J Respir Crit Care Med 2010; 181: 315-323. 
7 Wenzel SE, Busse WW. Severe asthma: lessons from the Severe Asthma Research Program. J Allergy Clin Immunol 2007; 119: 14-21.

8 ten Brinke A, Sterk PJ, Masclee AA, et al. Risk factors of frequent exacerbations in difficult-to-treat asthma. Eur Respir J 2005; 26: 812-818.

9 Department of Health, Gazdar C, Pettit R. Out in the Open: Breaking Down the Barriers for Older People. London, Department of Health, 2000.

10 Marsteller JA, Hsu YJ, Reider L, et al. Physician satisfaction with chronic care processes: a cluster-randomized trial of guided care. Ann Fam Med 2010; 8: 308-315.

11 Boyd CM, Reider L, Frey K, et al. The effects of guided care on the perceived quality of health care for multi-morbid older persons: 18-month outcomes from a cluster-randomized controlled trial. J Gen Intern Med 2010; 25: 235-242.

12 Boult C, Reider L, Leff B, et al. The effect of guided care teams on the use of health services: results from a cluster-randomized controlled trial. Arch Intern Med 2011; 171: 460-466.

13 Ross S, Curry N, Goodwin N. Case Management. What it is and How it Can Best be Implemented. London, King's Fund, 2011.

14 Hutt R, Rosen R, McCauley J. Case-managing Long-term Conditions. London, King's Fund, 2004.

15 Pond Z, Burke H, Duffus C, et al. Rising to the GINA Asthma Challenge: thinking beyond just asthma. Eur Respir J 2012; 40: 280-281.

16 Dinelli DL, Higgins JC. Case management of asthma for family practice patients: a pilot study. Mil Med 2002; 167: 231-234.

17 Schulte A, Musolf J, Meurer JR, et al. Pediatric asthma case management: a review of evidence and an experimental study design. J Pediatr Nurs 2004; 19: 304-310.

18 Ryan D, Murphy A, Ställberg B, et al. 'SIMPLES': a structured primary care approach to adults with difficult asthma. Primary Care Respir J 2013; 22: 365-273.

19 Abayaratne D, Babu S, McCulloch A, et al. Can the multidisciplinary input of an asthma nurse specialist and respiratory physician improve the discharge management of acute asthma admissions? Clin Med (Lond) 2011; 11: 414-415.

20 Thomas M, McKinley RK, Freeman E, et al. Breathing retraining for dysfunctional breathing in asthma: a randomised controlled trial. Thorax 2003; 58: 110-115.

21 Yorke J, Fleming SL, Shuldham CM. Psychological interventions for adults with asthma. Cochrane Database Syst Rev 2006; 1: CD002982.

22 Smith JR, Mugford M, Holland R, et al. A systematic review to examine the impact of psycho-educational interventions on health outcomes and costs in adults and children with difficult asthma. Health Technol Assess 2005; 9: 1-167.

23 Heaney LG, Conway E, Kelly C, et al. Prevalence of psychiatric morbidity in a difficult asthma population: relationship to asthma outcome. Respir Med 2005; 99: 1152-1159.

24 Fellows JL, Flower L, Blakey J, et al. Case series: the application of "third wave" cognitive behavioural therapies in difficult to treat asthma. J Asthma 2015; 52: 905-912.

25 Haldar P, Pavord ID, Shaw DE, et al. Cluster analysis and clinical asthma phenotypes. Am J Respir Crit Care Med 2008; 178: 218-224.

26 Patil VK, Townshend C, Mitchell F, et al. An outreaching model of tertiary difficult asthma care reduces adverse asthma outcomes and healthcare utilisation costs. Eur Respir J 2016; 47: 1857-1860. 